\title{
SER VÍCTIMA DE BULLYING HOMOFÓBICO EN URUGUAY LAS VOCES DE VARONES ADOLESCENTES DE MONTEVIDEO
}

\author{
Gonzalo Gelpi* \\ Universidad de la República, Uruguay \\ ggelpi@psico.edu.uy
}

Recibido: 8/10/2018 Aceptado: 21/11/2018

\section{Resumen}

En este trabajo se presentan resultados del proyecto de investigación "Bullying homofóbico y exclusión escolar en Ciclo Básico: Indagando en las experiencias de varones adolescentes de liceos públicos y privados de la ciudad de Montevideo". El mismo se ejecutó entre el 2016-2018. Se trató de un diseño metodológico cualitativo de tipo exploratorio que empleó el método de la Teoría Fundamentada. La muestra está compuesta por varones adolescentes de entre 12 y 19 años, residentes en Montevideo, víctimas de bullying homofóbico y que presentaron una interrupción temporal o definitiva de sus trayectorias educativas. Se realizaron 20 entrevistas en profundidad hasta alcanzar el principio de saturación teórica. Los principales resultados indican que las experiencias de los adolescentes fueron significadas como negativas. Hubo matices en sus estrategias de afrontamiento, lo que visibiliza la diversidad dentro de la propia diversidad sexual. En las instituciones existe discriminación y se dirige contra las personas que son percibidas como "diferentes". El bullying ocurre en todos los contextos, pero varía en sus modalidades. Los entrevistados lo sufrieron en los primeros años de la adolescencia. El fenómeno guarda una relación directa con el género, se encuentra en la base de las desigualdades de género. La no adhesión a las normas de género genera mayor rechazo en el entorno escolar que la orientación sexual homosexual. Los agresores solían ser varones con características similares, atributos asociados a la masculinidad hegemónica. En todos los casos ejercieron bullying directo y predominó la violencia verbal. La exposición a este fenómeno produjo diversos efectos bio-psico-sociales en los entrevistados.

Palabras clave: Enseñanza Media - Adolescencia - Bullying - Homofobia - Exclusión.

\section{Abstract}

This article presents the results of the investigation "Homophobic bullying and scholar dropout in High school: Knowing the experiences of male adolescents of public and private schools in Montevideo". The current investigation was carried on in the past two years. It consists of a qualitative, explorative-type methodology design based on Grounded Theory. The sample is composed of male adolescents aged between 12 and 19 years old, living in Montevideo, victims of homophobic bullying presenting a temporal or definitive interruption of their educative trajectory. 20 interviews were carried out until theoretic saturation was reached. The main results showed that the experiences of the adolescents were signified as negative. There were nuances in their coping strategies, which makes visible the diversity within the sexual diversity itself. In institutions there is

\footnotetext{
* Licenciado en Psicología, docente del Programa Género, Salud Reproductiva y Sexualidades de la Facultad de Psicología (UdelaR, Uruguay) y también docente del Programa de Posgrado en Género, Sociedad y Políticas de la Facultad Latinoamericana de Ciencias Sociales en Argentina. Diplomado en Ciencias Sociales con mención en Género y Políticas Públicas por la Facultad Latinoamericana de Ciencias Sociales en Argentina y se encuentra próximo a la defensa de su tesis de Maestría en Género, Sociedad y Políticas. También se desempeña como tallerista de sexualidad en diferentes centros educativos $p$ úblicos y privados de EnseñanzaMedia. Sus líneas de desarrollo académico son los estudios de género y diversidad sexual aplicados al ámbito educativo y con población adolescente.
} 
REVISTA de LA Escuela de CIENCIAS DE LA EdUCACIÓN, AÑo 15, NRO. 14, VOL. 1, ENERO A JUNIO DE 2019. PÁGINAS 36-44. ISSN 1851-6297 (DESDE DICIEMBRE DE 2006 A DICIEMBRE DE 2017). ISSN 2362-3349 (EN LINNEA). SER VICTIMA DE BULLYNG HOMOFÓBICO EN URUGUAY. LAS VOCES DE VARONES ADOLESCENTES DE MONTEVIDEO. GONZALO GELPI.

discrimination and it is directed against people who are perceived as "different". Bullying occurs in all contexts but varies in its modalities. Interviewees suffered it during their first years of adolescence. The phenomenon has a direct relationship with gender, due to the fact that it is recognized as the base of gender inequalities. Moreover, non-adherence to gender norms generates greater rejection in the school environment than homosexual sexual orientation. The aggressors used to be males with similar characteristics, attributes associated with hegemonic masculinity. In all cases, direct bullying and verbal violence predominated. Exposure to this phenomenon produced several biopsycho-social effects in the interviewees.

Keywords: Highschool - Adolescence - Bullying - Homophobia - Dropout.

\section{Introducción}

\subsection{Nociones generales sobre el concepto bullying}

Las discriminaciones, maltratos y abusos en el espacio escolar tienen un amplio historial. Sin embargo, los primeros estudios sobre bullying (categoría creada para observar al fenómeno) se produjeron en los países escandinavos en la década de los `70 (1) (Olweus, 1993). La palabra bullying es un anglicismo que se podría traducir como "matonismo" y se usa correctamente cuando la situación implica exclusivamente un acoso entre pares y en contexto escolar. En la academia no existe unanimidad para definir la problemática, hay varios enfoques teóricos y varía el modo de conceptualizarla.

Es importante diferenciar lo que es una situación puntual de violencia escolar de un caso de bullying. Para afirmar que un sujeto está padeciendo bullying se deben presentar una serie de características. Para Olweus (1993) las tres más importantes son las siguientes: a) recibir conductas con intencionalidad de causar daño sin motivo aparente (burlas, amenazas, insultos, intimidaciones, agresiones físicas, agresiones sexuales, aislamiento sistemático, ruptura de materiales y robos), b) que se prolonguen en el tiempo siendo ataques sistemáticos (a pesar de que no hay unanimidad sobre el período de tiempo mínimo), c) que suponga un abuso de poder basado en relaciones asimétricas.

Dan Olweus (1993) nos ofrece pensar el fenómeno desde la lógica del triángulo del bullying. Desde la perspectiva del autor, dicho triángulo, es conformado por la víctima, el/los agresor/es y los espectadores (bystanders). Se destaca que puede haber espectadores tanto activos como pasivos. Los activos se caracterizan por reforzar la conducta del/los agresor/es mediante comentarios, aplausos, filmaciones, entre otras acciones. Por otro lado, los pasivos, suelen quedar al margen de la escena de acoso, pero pese a no estar de acuerdo con las agresiones, silencian lo que ocurre, lo que coopera a reproducir las violencias (Gómez et al. 2007 y Domínguez, 2011).

Es interesante interrogarse en este esquema por el papel y la responsabilidad de las instituciones educativas, ya que, la mayoría de las acciones se cometen dentro de los edificios escolares y, con este abordaje, se invisibiliza a la escuela. En esta línea, es posible afirmar que la dinámica del bullying suele ser más amplia, cuenta con la convivencia de otros actores del sistema (particularmente de los docentes, quienes son los referentes adultos más directos del estudiantado dentro de las escuelas) (Observatorio Argentino de Violencia en las Escuelas, 2011).

El bullying se puede aplicar de un modo directo y/o indirecto. El directo es cuando la víctima sufre de agresiones físicas, verbales y/o sexuales, mientras que, el indirecto se caracteriza por la presencia de rumores, chismes, exclusiones, rotura de materiales, robo de pertenencias, extorsiones económicas, entre otros (Olweus, 1993). Las situaciones suelen implicar múltiples violencias, las más frecuentes son: física, verbal, social, patrimonial y/o sexual (Gómez et al. 2007). En todas ellas, la violencia psicológica está presente. Es importante señalar que los tipos de violencias dialogan y se retroalimentan entre sí.

Ruiz (2009) enumera una serie de condicionantes para padecer bullying e identifica de ese modo a los grupos blancos de las agresiones, entre ellos se encuentran: estudiantes con sobrepeso, autoidentificados como LGBT, con alguna discapacidad física y/o intelectual o que pertenecen a una minoría étnico/racial o religiosa. Estudios internacionales destacan que no existen diferencias acentuadas por el nivel socioeconómico y que con frecuencia se presentan casos tanto en el ámbito educativo público como privado. La franja etaria de mayor riesgo para padecer bullying es la comprendida entre los 11 y 14 años (Domínguez, 2011). Igualmente, no se debe descuidar que el bullying, afecta a todos los actores de la comunidad educativa, sus variables y expresiones (desde las atrozmente virulentas a las más simbólicas) son violatorias de los derechos humanos y de principios como la igualdad y no discriminación. Además, obstruye los procesos de enseñanza - aprendizaje y produce efectos negativos en la esfera de lo bio-psico-social de los implicados, especialmente en las víctimas.

Desde su surgimiento hasta la actualidad, el bullying, ha sido objeto de estudio de varias disciplinas y mediante diversas metodologías en todas las culturas. Hay evidencia empírica de que el fenómeno ocurre en todo el mundo. Los antecedentes existentes mayoritariamente se han ocupado de indagar la fenomenología del bullying, las relaciones con otros fenómenos sociales, la vinculación con ciertas patologías, el impacto psicológico y emocional en los distintos actores involucrados - especialmente en las víctimas - y la efectividad de las prácticas antibullying.

Además, recientemente, surgió un nuevo problema a atender, el cyberbullying (2), fenómeno que se retroalimenta con el bullying tradicional. Se debe destacar que muy especialmente en las últimas tres décadas, se produjo una expansión del uso del concepto bullying a nivel global. Este campo de estudios 
REVISTA de LA ESCUela de CIENCIAS DE LA EdUCACIÓN, AÑo 15, NRO. 14, VOL. 1, ENERO A JUNIO dE 2019. PÁGINAS 36-44. ISSN 1851-6297 (DESDE DICIEMBRE DE 2006 A DICIEMBRE DE 2017). ISSN 2362-3349 (EN LINEA). SER VICTIMA DE BULLYNG HOMOFÓBICO EN URUGUAY. LAS VOCES DE VARONES ADOLESCENTES DE MONTEVIDEO. GONZALO GELPI.

se consolidó y paulatinamente se fueron diseñando dispositivos de prevención e intervención y distintas políticas públicas antibullying (Olweus, 2004).

\subsection{Algunas particularidades del bullying homofóbico}

Los estudios sobre bullying homofóbico son aún más recientes, los primeros registros se ubican en la década de los '90. Los países referentes internacionalmente son Gran Bretaña, Estados Unidos y en habla hispana destaca España (Generelo, 2012; Ruiz, 2009). El bullying homofóbico tiene su origen en los valores de la "masculinidad" y "feminidad" que se dan por supuestos en nuestra sociedad, "se ataca a aquellos sujetos que transgreden las normas socialmente pautadas de comportamiento para hombres y mujeres" (Ruiz, 2009, p. 93). Según Jennett (2004) "el bullying homofóbico puede presentarse en diferentes formas incluyendo burlas, insultos y ridiculización, rumores, intimidación, empujones, golpes, robos o destrucción de pertenencias, marginación social, acoso cibernético, agresión física o sexual y amenazas de muerte" (Jennett, 2004, p. 20).

Este tipo de acoso se sustenta en la orientación sexual, identidad de género y/o expresión de género de los sujetos. Frecuentemente lo suelen padecer estudiantes autoidentificados como gays, bisexuales o lesbianas, estudiantes trans, estudiantes a los cuales se les asigna una orientación no heterosexual por mantener comportamientos alejados de las normas de género vigentes en una sociedad en determinado contexto socio-histórico, estudiantes que son integrantes de una familia monoparental y amigos/as de la víctima mediante lo que se conoce como "transferencia del estigma" (Ruiz, 2009).

En los últimos años los diferentes organismos internacionales le han insistido a los Estados que deben reforzar el compromiso en el combate contra el bullying (homofóbico) porque este viola derechos humanos fundamentales y produce efectos muy nocivos en la comunidad educativa (UNESCO, 2018; CIDH, 2015; UNESCO, 2015; CEPAL, 2013; UNESCO, 2013; UNICEF, 2011; ONU, 2006). Pese a las recomendaciones antes expuestas y a los avances legales y sociales que tuvo Uruguay en materia de género y diversidad sexual entre los años 2005-2015, en la actualidad, aún no existe ningún instrumento para prevenir, detectar e intervenir ante situaciones de bullying por cualquier motivo incluyendo el basado en la homofobia. Por distintas razones, el diseño de mecanismos efectivos en la lucha contra la homofobia fue el gran pendiente de un período marcado por logros para el colectivo de personas LGBT (Sempol, 2013a; 2013b).

A pesar de que Uruguay es reconocido internacionalmente por ser un país gay friendly porque tiene normativas que reconocen la igualdad de derechos de las personas LGBTI, en la vida cotidiana, este colectivo, aún sufre la homofobia. Uno de los espacios donde ésta más se manifiesta es en el cotidiano escolar, por esa razón, el presente estudio se centró en explorar la incidencia del bullying homofóbico en la exclusión escolar de varones adolescentes de la ciudad de Montevideo en Enseñanza Media. Además, en general, los antecedentes nacionales de investigación sobre bullying son escasos debido a que la problemática ingresó a la agenda de investigación recientemente (Aristimuño y Noya, 2015; MIDES INJU, 2015; UNICEF -CES, 2013).

Aunque es difícil medir la prevalencia del bullying homofóbico porque no todas las personas que lo sufren son LGBT ni todas las personas autoidentificadas como LGBT lo padecen ni lo informan, Ovejas Negras y GLSEN (2017) coordinaron una Encuesta Nacional de Clima Escolar específica para estudiantes LGBT que permitió aproximarse a las experiencias de este colectivo. De dicho estudio participaron 423 adolescentes provenientes de todos los departamentos del país y que al momento de la encuesta tenían entre 13 y 20 años. Los resultados mostraron que, los adolescentes LGBT se encuentran en desigualdad de condiciones con relación a sus pares heterosexuales, están expuestos a preconceptos y mensajes negativos, discriminación y exclusión. De todos modos, se debe mencionar que, en los últimos años, los cambios legales propiciaron de marco habilitante para introducir la diversidad sexual en los centros educativos bajo la legitimación que le confería el ser objeto de estudio, de legislación y estar en la agenda pública (Schenck, 2014).

\section{Método}

Se trató de un estudio cualitativo de tipo exploratorio que empleó el método de la Teoría Fundamentada. El alcance del estudio fue descriptivo. Se definió una muestra intencional teórica (no probabilística) sustentada en el principio de saturación teórica de la Teoría Fundamentada. La técnica empleada para recolectar la información fue la entrevista en profundidad. De la investigación participaron 20 adolescentes varones de entre 12 y 19 años, residentes en Montevideo, de diferentes contextos socioeconómicos, que habían padecido bullying en instituciones educativas públicas o privadas por motivos relativos a la orientación sexual y/o expresión de género y que habían interrumpido temporal o definitivamente sus trayectorias educativas.

Estos criterios de inclusión se consideraron como los más oportunos por varios aspectos, entre los más importantes: porque se contempla a todos los adolescentes en edad de cursar la Enseñanza Media, tanto aquellos que pasaron la discriminación y/o acoso hace algún tiempo como quienes lo estaban padeciendo en la actualidad, porque se respeta la dimensión subjetiva de los participantes y no son los investigadores quienes definen si el sujeto padeció bullying o no y además porque no es excluyente que hayan retomado sus trayectorias educativas luego de los episodios de discriminación y/o acoso. Al tratarse de una convocatoria más abierta, el material resultante de las entrevistas fue más amplio y arrojó información sobre otros fenómenos que sufren los adolescentes LGBT en el cotidiano escolar y que afectan su salud. 
ReVista de LA Escuela de Ciencias de LA EdUCACIÓn, AÑo 15, NRO. 14, VOL. 1, ENERO A JUNIO de 2019. PÁgINAS 36-44. ISSN 1851-6297 (DESDE DICIEMBRE DE 2006 A DICIEMBRE DE 2017). ISSN 2362-3349 (EN LINNEA). SER VICTIMA DE BULLYNG HOMOFÓBICO EN URUGUAY. LAS VOCES DE VARONES ADOLESCENTES DE MONTEVIDEO. GONZALO GELPI.

La codificación del material se hizo mediante método inductivo. Se resalta que se cumplió con todas las consideraciones éticas inherentes a tareas investigativas con seres humanos en Uruguay.

\section{Resultados}

Primeramente se debe aclarar que, para el presente estudio, se optó por la categoría bullying porque es un concepto ampliamente utilizado en el mundo y ha dominado los discursos sobre las violencias en el ámbito educativo. En sus orígenes, dicho concepto cooperó para desnaturalizar prácticas cotidianas del currículo informal de las instituciones educativas y puso en debate que en ocasiones las escuelas no son lugares seguros para los estudiantes (Observatorio Argentino de Violencias en las Escuelas, 2011). A su vez, actualmente el bullying (homofóbico) es parte de la agenda de los organismos internacionales y objeto de políticas públicas en los diferentes países.

Se debe destacar que, en la última década, el concepto bullying ha permeado en la realidad local y los propios adolescentes de todos los contextos lo han adoptado para su uso cotidiano. La mediatización del concepto en ocasiones cooperó para desvirtuarlo o banalizarlo porque cuando todo es bullying nada lo es y se corre el riesgo del vaciamiento de la categoría (MSP, 2017).

Al momento del estudio, la mayoría de entrevistados tenía entre 16 y 19 años, esto demuestra una vez más lo complicado de encontrar testimonios de adolescentes menores de 15 años autoidentificados como gays o bisexuales. Principalmente por un motivo: los adolescentes que suelen participar de proyectos de investigación sobre diversidad sexual ya tienen asumida su orientación sexual y la correspondiente identidad sexual en los distintos espacios por los cuales circulan (Savin - Williams, 2009). A su vez, aunque gran parte de los adolescentes asistían al sistema público de educación, el número de entrevistas realizadas a quienes tenían experiencias en ambos sectores o que habían sido escolarizados exclusivamente en el sector privado no fue despreciable, por lo que, ambas realidades estuvieron representadas en los testimonios.

En cuanto a los resultados, las experiencias de los adolescentes entrevistados para este estudio relativas al bullying homofóbico fueron significadas como negativas. En este trabajo se entiende a la experiencia desde los abordajes que toman en cuenta "las vivencias y los sentidos y que reconocen la capacidad de los sujetos de reconstruir significados, ya sea a través de ponerlos en acción o de narrar su experiencia, es decir, que se colocan desde la dimensión subjetiva" (Guzmán y Saucedo, 2015, p.1023). Para Dewey (2004) se trata de una unidad de análisis que toma en cuenta las interacciones entre las personas y el contexto, las cuales se determinan mutuamente, es decir, tienen un carácter transaccional. La noción de experiencia se abre a los afectos y a las emociones (Guzmán y Saucedo, 2015). Como lo señala Roth (2014), las experiencias se construyen en el interjuego continuo entre lo práctico, lo intelectual y lo afectivo.

En los relatos de los adolescentes hubo matices porque dichas experiencias fueron integradas, interpretadas y resignificadas de distinto modo, eso visibilizó la diversidad dentro de la propia diversidad sexual. Los adolescentes presentaron dificultades para transmitir y compartir lo que les ocurrió. Según Viñar (2009) el horror y el dolor extremo no generan experiencia sino espanto, no genera representaciones y relato sino vacío representacional y por consiguiente lo ocurrido es difícilmente transmisible y compartible. Tras escuchar los relatos, se puede concluir que, aún hay horrores impronunciables, se accedió a lo que estaban en condiciones de dar a conocer. Por estos motivos, la metáfora de la punta del iceberg es válida para explicar el fenómeno del bullying por cualquier causa.

Todos los casos se produjeron en el nivel de Ciclo Básico de Enseñanza Media, o sea, aproximadamente entre los 11 y los 15 años. Este dato coincide con la literatura internacional (Domínguez, 2011; Generelo, 2012; Ruiz, 2009). Al parecer, el bullying homofóbico, suele producirse mayoritariamente en la adolescencia temprana y media, por lo que, es posible identificar una franja etaria de riesgo también a nivel nacional.

Al consultarles por qué creían que habían sido discriminados y/o acosados, los entrevistados manifestaron que probablemente hubiese sido por ser "diferentes" al resto, especialmente por ser homosexuales o por no cumplir con las normas de género vigentes, es decir, porque sus expresiones de género eran discordantes con lo que se espera socialmente de un varón.

Creo que por eso, por la forma de hablar, por la forma de caminar, relacionarme con mujeres, creo que eso es crucial que un varón rodeado de mujeres siempre, todo el tiempo es raro. En las clases de gimnasia yo nunca jugaba al futbol, siempre al vóley (Sujeto 3).

Un conjunto de adolescentes se cuestionaba qué habían hecho para recibir las agresiones, no entendían el trato desigual que recibieron de sus compañeros e intentaban justificarlos apelando a racionalizaciones. Ellos no terminaban de comprender lo que sucedía y, la asimilación e integración de las experiencias relativas al bullying, por momentos, se daba de modo parcial. Esta situación también les producía tristeza, cansancio, rabia, ira, desazón y soledad.

Muchas veces me acostaba y si... Iloraba y me preguntaba ¿por qué? me hacen esto ¿por qué yo tengo que soportarlo, por qué soy el único en la clase que le hacen esto...? (Sujeto 20).

En pasajes de entrevistas, algunos adolescentes se representaron a ellos mismos como culpables de lo que les había sucedido. En estos casos, había una interiorización del discurso de los agresores, ellos mismos se posicionaban desde un paradigma culpabilizador para analizar y narrar sus propias experiencias. Dichos relatos se relacionaban con mayores niveles de homofobia interiorizada. A partir de la exposición constante a un orden profundamente heteronormativo, este conjunto de entrevistados tenía 
REVISTA de LA ESCUela de CIENCIAS DE LA EdUCACIÓN, AÑo 15, NRO. 14, VOL. 1, ENERO A JUNIO dE 2019. PÁGINAS 36-44. ISSN 1851-6297 (DESDE DICIEMBRE DE 2006 A DICIEMBRE DE 2017). ISSN 2362-3349 (EN LINEA). SER VICTIMA DE BULLYNG HOMOFÓBICO EN URUGUAY. LAS VOCES DE VARONES ADOLESCENTES DE MONTEVIDEO. GONZALO GELPI.

representaciones predominantemente negativas sobre la homosexualidad.

Yo sentía una culpa muy grande y siempre fui muy depresivo por ese aspecto ¿entendés? Era como que me sentía un mal hijo o una mala persona porque me tocó ser así y me merecía lo que me pasaba (Sujeto 11).

Asimismo, en ocasiones, ese discurso culpógeno, se vinculaba con los efectos negativos producidos por la primera intervención institucional tras la denuncia del caso. Este punto reafirma lo sostenido por Piñuel y Oñate (2006). Ellos desarrollan características como elementos típicos de la dinámica del bullying a nivel institucional: 1) síndrome de negación de la institución (banalización y trivialización de la violencia); 2) error básico de atribución (la víctima es responsable de lo que ocurre); 3) proceso de victimización secundaria del acosado, introyección de la culpa (indefensión aprendida); 4) pacto de silencio y pacto de mutua indiferencia.

Por otro lado, algunos entrevistados resaltaron que el bullying homofóbico comenzó en las redes sociales online y posteriormente se trasladó a los edificios escolares. En otros casos, el acoso comenzó dentro de los edificios escolares y luego se expandió a las redes sociales online. En el marco de la dinámica del acoso, el uso de las TIC para cometer agresiones intensificó algunos sentimientos negativos en las víctimas a causa de la sobreexposición a mensajes ofensivos. Desde las experiencias de los entrevistados se puede afirmar que, el fenómeno del bullying se retroalimenta del cyberbullying y viceversa. A su vez, las redes sociales online se configuran como otro espacio de vigilancia, control, normalización y castigo hacia quienes se alejan de las normas de género vigentes en nuestra cultura. En dichos espacios se reeditan las lógicas de exclusión hacia ciertos sujetos, cuerpos e identidades y, se producen y reproducen desigualdades de género.

Aunque no hubo unanimidad en cuanto a la sistematicidad de las agresiones tanto presenciales como virtuales, la mayoría de los relatos coinciden en que, el primer semestre de clases suele ser especialmente duro porque casi todos los días sufrían ataques. Del material se desprende que los entrevistados desplegaron diferentes estrategias de afrontamiento para combatir al bullying dependiendo de sus recursos psíquicos y de sus características de personalidad.

Se debe resaltar que en todos los casos hubo un período de tiempo, al inicio de la dinámica del bullying, donde mecanismos psicológicos como la negación y la desmentida estuvieron muy activos. Estos limitaron la capacidad de acción de estos adolescentes, tanto para enfrentar la situación por ellos mismos como para pedirle ayuda a terceros.

A partir de las situaciones displacenteras a las que se enfrentaron cotidianamente pusieron en juego diferentes recursos para sobrevivir en la institución y no interrumpir definitivamente sus trayectorias educativas. Salvo excepciones, quienes sufrieron la exclusión escolar, luego de un tiempo se reinsertaron nuevamente en el sistema educativo formal. Estos adolescentes lograron identificar como espacios peligrosos para su integridad a los recreos, el patio, la cantina, los paseos y las clases de gimnasia. En esos sitios ellos se sentían vulnerables, en especial por la falta de control del mundo adulto. El solo hecho de detectar esos lugares como amenazantes, como peligrosos y comenzar a evitarlos, ya configura en sí una estrategia de afrontamiento al problema. A su vez, esta aclaración también es un modo de evitar reducirlos exclusivamente a la figura de "víctima pasiva".

Al tratarse de situaciones que mayoritariamente implican un abuso de poder o una fuerza desigual real o percibida, los adolescentes manifestaron no sentirse fuertes como para confrontar directamente con sus agresores y generalmente quienes salían en su defensa eran un grupo de pares muy pequeño conformado casi siempre exclusivamente por mujeres. Sin embargo, algunos adolescentes se sobrepusieron a la adversidad y consiguieron que las agresiones cesaran, por ejemplo, al agredir físicamente a sus victimarios, lo que configuró un hito para romper con la dinámica del bullying. Este punto también puede ser abordado desde los aportes de los estudios de las masculinidades porque pareciera que los agresores necesitaron que hablaran su propio lenguaje, un lenguaje en clave masculina que les permitió a las víctimas ser reconocidas por sus pares como varones, desarticulando la asociación gay-femenino. Algo así como una demanda de demostración de virilidad, una necesidad imperiosa para calmar la angustia que les despierta el enfrentarse a una masculinidad que no adhiere a los atributos hegemónicos (Badinter, 1993).

A partir del material obtenido se puede concluir que, en todos los casos, los agresores eran varones con similares características, atributos asociados a la masculinidad hegemónica. Por otro lado, los espectadores pasivos eran mujeres, mientras que, los espectadores activos eran varones.

Las experiencias de estos adolescentes condicen con la literatura internacional, la cual sostiene que suele haber más agresores y más víctimas entre los varones, que los varones suelen ser agredidos solo por otros varones que aplican el bullying directo y que los casos que implican varones suelen ser los más graves. En cambio, las mujeres suelen practicarles bullying a otras mujeres y de tipo indirecto, haciendo uso predominantemente de la violencia social y verbal (Gómez et al. 2007). Por estos motivos es posible afirmar que la problemática se encuentra en la base de las desigualdades de género y afecta diferencialmente a varones y mujeres. Incluso, existen diferencias que se traducen en desigualdades entre los propios varones y las propias mujeres. Esto depende de la orientación sexual, identidad de género y expresiones de género de los estudiantes. Al parecer, ellos y ellas suelen ejercer la violencia en sintonía con los mandatos de género y los modelos hegemónicos de feminidad y masculinidad.

De hecho, los límites de género descriptos por Guasch (2007) son claros al expresar que la 
REVISTA de LA Escuela de CIENCIAS de LA EdUCACIÓN, AÑo 15, NRO. 14, VOL. 1, ENERO A JUNIO de 2019. PÁGINAS 36-44. ISSN 1851-6297 (DESDE DICIEMBRE DE 2006 A DICIEMBRE DE 2017). ISSN 2362-3349 (EN LINEAA). SER VICTIMA DE BULLYNG HOMOFÓBICO EN URUGUAY. LAS VOCES DE VARONES ADOLESCENTES DE MONTEVIDEO. GONZALO GELPI.

homosexualidad interpela de distinto modo a varones y a mujeres. Es especialmente a ellos que les preocupa demostrar constantemente que no son homosexuales, o sea, que son verdaderamente "machos". En los primeros años de la adolescencia, los varones, sienten la necesidad de mostrarse ante sus pares como "machos" para afianzar su identidad masculina y tener la aceptación del grupo, esa necesidad, puede desencadenar una etapa violentamente homofóbica. La producción de heterosexualidad es acompañada por el rechazo de la homosexualidad (Cornejo, 2010; Olavarría, 2005).

Por esa razón podría afirmarse que en ocasiones el bullying homofóbico surge a partir de una amenaza subjetiva que vivencian ciertos varones en lo que refiere a la fragilización de sus identidades de género. El temor a lo femenino y a la homosexualidad es un núcleo constitutivo de la identidad de género masculina y alcanza su punto máximo durante la adolescencia, aunque especialmente durante la adolescencia ese temor convive con elementos altamente homoeróticos (Burin y Meler, 2009). Los varones con atributos asociados a la masculinidad hegemónica suelen ser los principales cometedores de conductas homofóbicas y también por miedo a la transferencia del estigma suelen evitar todo contacto con sujetos que no sean reconocidos por el entorno como "machos verdaderos" (Ruiz, 2009). Las agresiones suelen padecerlas mayoritariamente los varones autoidentificados como gays, bisexuales o que presentan una expresión de género que no se adecua a lo esperado socialmente (López Amurrio, 2013; Cornejo, 2010; García, 2010; Ruiz, 2009; Olavarría, 2005).

El varón busca su masculinidad y obviamente si hay otro varón que es femenino le va a servir para ir y compararse directamente y sentirse bien y le da el derecho a opinar y cagarse de la risa (Sujeto 11).

La mayoría de los adolescentes entrevistados sufrió bullying directo. Los tipos de violencia dialogan entre sí y se retroalimentan. La violencia psicológica es transversal y está presente en todos los otros tipos. Dentro de la tipología de las violencias, la más mencionada por los adolescentes fue la verbal. Sin embargo, aunque en menor medida, los entrevistados también informaron haber sufrido agresiones físicas, patrimoniales y en dos casos inclusive violencia sexual.

Me decían puto, gay, trolo, traga... todo, te hacían quedar re mal porque te re humillaban. Te humillaban y vos no sabías qué hacer si pegarles, si no pegarles, si reírte con ellos, dejar de reír y llorar (Sujeto 6).

Cuando entré al liceo fue horrible. En primer año me acuerdo que pasaba llorando todo el día porque no quería ir al liceo. Me ponían carteles en la espalda, tipo me dibujaban penes, me ponían gordo puto y ta, pasaba llorando (Sujeto 13).

En la adolescencia temprana, se comienza a hacer uso de insultos propios del mundo adulto. El dominio de un código verbal obsceno y su puesta en funcionamiento se legitima mayormente si es por parte de los varones, es un elemento de la gramática masculina. Este acontecimiento permite tejer complicidades e identidades, poniéndose en juego la pasividad y actividad, en tanto papeles sexuales y sociales (Fachel Leal en Valdés y Olavarría, 1998). El uso de insultos para diferenciarse de lo construido como lo diferente, lo no hegemónico, los otros, es necesario en la medida que la identidad de género de los varones se construye en base a un modelo dual que comporta potencia y carencia; es decir, provee el privilegio del dominio, pero a la vez queda condenado a demostrar constantemente "su derecho" a tal privilegio (Olavarría, 2005).

La violencia patrimonial se presentó con mayor asiduidad en el sector privado. La violencia física se informó en escasas ocasiones. Desde la perspectiva de los entrevistados esto se debe a que por lo general las sanciones institucionales suelen ser fuertes. En cambio, las otras violencias, salvo excepciones, no conllevan ningún castigo. De todos modos, también hubo relatos donde se manifestó que ante agresiones físicas tampoco hubo intervenciones desde el mundo adulto ni sanciones a los agresores.

Las experiencias de bullying homofóbico produjeron efectos bio-psico-sociales negativos en diferentes dimensiones de la vida de los adolescentes entrevistados. Es importante resaltar que no todos los adolescentes tuvieron los mismos efectos ni con la misma intensidad ni constancia. Cada uno procesó sus experiencias con los recursos que contaban en ese momento y dependiendo de diversos factores que exceden los objetivos de este estudio. En general los adolescentes desarrollaron distintas sintomatologías, las cuales perduraron más o menos en el tiempo.

En los relatos se constataron afectaciones en el estado de ánimo, en la autoestima, en los ciclos de sueño, en los hábitos alimenticios, en el rendimiento académico, en los niveles de concentración y atención dentro y fuera de clase. Además se produjo un debilitamiento de sus redes sociales que desencadenó en situaciones de exclusión escolar. Dicha exclusión se produjo en dos sentidos, la promovida por los pares, pero también la motivada por la propia institución educativa. La escuela en tanto institución disciplinar refuerza la heteronormatividad. Las prácticas escolares cotidianas producen clasificaciones, jerarquizaciones y marginalizaciones que comprometen la garantía al derecho a la educación de calidad (Junqueira, 2013).

No me levantaba. En ese tiempo iba al club y lo dejé. No comía y vivía encerrado en el cuarto, nada más (Sujeto 1).

En esa época tenía miedo a engordar por si me seguían discriminando, entonces no comía, o sea, rocé casi la anorexia. Tenía miedo de que me discriminaran por más cosas y como tenía inseguridad dejé de comer (Sujeto 6). 
REVISTA de LA ESCUela de CIENCIAS DE LA EdUCACIÓN, AÑo 15, NRO. 14, VOL. 1, ENERO A JUNIO dE 2019. PÁGINAS 36-44. ISSN 1851-6297 (DESDE DICIEMBRE DE 2006 A DICIEMBRE DE 2017). ISSN 2362-3349 (EN LINEA). SER VICTIMA DE BULLYNG HOMOFÓBICO EN URUGUAY. LAS VOCES DE VARONES ADOLESCENTES DE MONTEVIDEO. GONZALO GELPI.

\section{Dormía poco, me tenía que obligar a dormir porque sino no dormía (Sujeto 3).}

Sus redes sociales se vieron empobrecidas porque al llegar a sus casas no querían salir de sus habitaciones, no mantenían conversaciones prolongadas con sus padres y otros familiares e inclusive interrumpieron actividades placenteras que realizaban generalmente por fuera de las instituciones educativas.

Durante los momentos críticos algunos estaban más depresivos y dormían más, otros más ansiosos y sufrieron de insomnio, algunos dejaron de comer y otros a partir del bullying comieron con mucha mayor frecuencia. Asimismo, les preocupaba sobremanera tener más elementos que les colocara en el grupo de los que no cumplen con la "norma", por esa razón, tenían un cuidado excesivo por la estética y desplegaban diversas estrategias para controlar lo que les era posible controlar, otras cosas al tratarse de atributos estructurales les excedía. A partir de estos elementos es posible afirmar que, la noción de normalidad es estructurante del psiquismo. Los adolescentes invierten mucha energía psíquica en producir normalidad y cuando no cumplen con lo esperado se manifiesta cierto malestar psíquico. Igualmente, siempre se debe de considerar la tensión existente entre la producción social de normalidad y los parámetros de normalidad de cada sujeto (Bergeret, 1980). A su vez, la interseccionalidad en tanto herramienta analítica se vuelve útil para comprender las experiencias singulares de estos sujetos en los cuales habitan múltiples identidades sociales productoras de privilegios y opresiones incluso simultáneamente.

En los momentos de mayor intensidad de las agresiones varios entrevistados se cuestionaron como posibilidad la autoeliminación. Quienes solo tuvieron ideaciones suicidas, dijeron no haberlo hecho solamente por no producirle sufrimiento a sus familiares. Este hecho no es menor porque el ser LGBT es un condicionante de la salud y un factor de riesgo para cometer suicidio- la tasa es mucho mayor si se compara con la de adolescentes heterosexuales- (López y Gelpi, 2015).

Ojalá nunca hubiese existido. Ahí fue el peor momento que me sentí. Igual creo que no he llegado al

límite de suicidarme, pero estuve cerca (Sujeto 9).

Varias veces pensé en matarme, pero no lo hice porque no quería generarle dolor a mamá, pero por ahí pasaba toda la noche pensando cómo sería mi velorio, todo (Sujeto 14).

Es menester señalar que todo el material obtenido permite identificar a la sexualidad en la base de los procesos de salud-enfermedad. Además, se debe resaltar que, los componentes de lo "enfermo", de lo "patológico", no estaban en los sujetos entrevistados ni en sus orientaciones sexuales, sino por el contrario, fue el contexto escolar y el entorno familiar que acabaron generando la mayoría de malestar psíquico en estos adolescentes. Todo lo antes expuesto incrementó los niveles de homofobia interiorizada en estos adolescentes. Meyer (2003) explica este fenómeno en base a una socialización temprana caracterizada por la heteronormatividad y por la exposición constante a actitudes homófobas que también hace que en algunos casos las propias personas autoidentificadas como LGBT interioricen el modelo hegemónico de sexualidad.

\section{Consideraciones finales}

Existe discriminación y bullying en todos los contextos socioeconómicos, pero hay variaciones en las modalidades de expresión. Generalmente se dirige contra las personas percibidas como "diferentes" o a quienes se les asignan atributos propios de grupos no hegemónicos. A partir de los relatos es posible identificar una franja etaria de riesgo para sufrir bullying homofóbico y es la comprendida entre los 11 y 15 años. La información recogida en cuanto a la sistematicidad de las agresiones se caracteriza por su heterogeneidad, no hay unanimidad, pero por lo general, los adolescentes identifican el primer semestre de clases como el más intenso y displacentero.

El fenómeno del bullying homofóbico se encuentra en la base de las desigualdades de género. La no adhesión a las normas de género genera mayor rechazo que la orientación sexual homosexual a la interna de los edificios escolares. En todos los casos, los agresores solían ser varones con atributos asociados a la masculinidad hegemónica, dichos varones ejercieron bullying directo. Los espectadores activos eran varones y los pasivos mujeres. Los entrevistados informaron que predominó la violencia verbal, a pesar de que se tiene evidencia empírica de otros tipos de violencias.

A partir de este estudio se constata una vez más la relación entre la construcción de una identidad de género masculina con atributos hegemónicos y la prevalencia de conductas homofóbicas, especialmente durante la adolescencia porque la homosexualidad interpela de distinto modo a varones y a mujeres. Entonces, algunos varones, al sentir una amenaza de fragilización de sus identidades de género, agreden a pares que desafían las normas de género vigente al tener expresiones de género disidentes.

Las experiencias de bullying homofóbico produjeron efectos bio-psico-sociales negativos en diferentes dimensiones de la vida cotidiana de los adolescentes entrevistados. Gran parte de los efectos coinciden con lo ya relevado por otros académicos a nivel internacional y regional. En próximos estudios, sería pertinente indagar las experiencias de otros actores con el fin de comprender a lo que se enfrentan los adolescentes autoidentificados como LGBT en el cotidiano escolar en otros contextos. Tales actores podrían ser: agresores, docentes, adolescentes gays y bisexuales del interior del país y adolescentes lesbianas y trans de Montevideo. Todo lo cual, nos permite ampliar el objeto de estudio y abordarlo desde la categoría bullying LGBTfóbico. 
REVISTA de LA ESCUela de CIENCIAS de LA EdUCACIÓN, AÑo 15, NRO. 14, VOL. 1, ENERO A JUNIO de 2019. PÁGINAS 36-44. ISSN 1851-6297 (DESDE DICIEMBRE DE 2006 A DICIEMBRE DE 2017). ISSN 2362-3349 (EN LINNEA). SER VICTIMA DE BULLYNG HOMOFÓBICO EN URUGUAY. LAS VOCES DE VARONES ADOLESCENTES DE MONTEVIDEO. GONZALO GELPI.

\section{Notas bibliográficas}

(1) En 1982 una tragedia obliga a las autoridades de Noruega a prestarle atención a la problemática. Tres jóvenes noruegos de entre 10 y 14 años se suicidaron, y las principales hipótesis sostenían que fue consecuencia del grave acoso que sufrían por parte de sus compañeros (Olweus, 1993).

(2) Consiste en una nueva modalidad de bullying, sus particularidades exceden el objetivo de este artículo. A su vez, tampoco existe unanimidad para denominar al fenómeno. Los motores de búsqueda pueden arrojar resultados con otras opciones, como, por ejemplo: ciberacoso, acoso cibernético, electrónico, digital y siempre entre iguales. Belsey (2006) conceptúa el cyberbullying como "el uso vejatorio de algunas tecnologías de la información y la comunicación (TIC), como el correo electrónico, las redes sociales, los mensajes del teléfono móvil, la mensajería instantánea, los sitios personales, y/o el comportamiento personal en línea difamatorio, de un individuo o un grupo, que deliberadamente, y de forma repetitiva y hostil, pretende dañar a otra persona" (p.235). Quienes deseen profundizar, pueden indagar los artículos de Maite Garaigordobil (referente de la temática en habla hispana).

\section{Referencias bibliográficas}

- $\quad$ Aristimuño, A. y Noya, J.C. (2015). "La convivencia escolar y el fenómeno del bullying en la enseñanza secundaria”. Páginas de Educación, 8(2), pp. 1-29.

- $\quad$ Badinter, E. (1993). XY: La identidad masculina. Alianza Editorial, Madrid.

- Belsey, B. (2006). Cyberbullying: An emerging Threat to the always of generation. Recuperado: http://www.cyberbullying.ca/pdf/Cyberbullying_Article_by_Bill_Belsey.pdf

- $\quad$ Bergeret, J. (1980). La personalidad normal y patológica. Gedisa, Barcelona.

- Burin, M. y Meler, I. (2009). Varones. Género y subjetividad masculina. Librería de Mujeres Editoras, Buenos Aires.

- Comisión Económica para América Latina y El Caribe (CEPAL). (2013). Consenso de Montevideo sobre Población y Desarrollo. CEPAL, Santiago.

- Comisión Interamericana de Derechos Humanos (CIDH). (2015). Violencia contra personas LGBTI. CIDH, Washington.

- $\quad$ Cornejo, J. (2010). “Jóvenes en la encrucijada”. Última década, 18(32), pp. 173 - 189.

- $\quad$ Dewey, J. (2004). Experiencia y Educación. Editorial Biblioteca Nueva, Madrid.

- Dominguez, F. (2011). "Las manifestaciones del bullying en adolescentes". Uaricha Revista Psicología (Nueva época), 8(17), pp. 19 - 33.

- Fachel Leal, O. (1998). "Sexualidad e identidad masculina: Impases y perspectivas de análisis". En T. Valdés y J. Olavarría (eds.), Masculinidades y equidad de género en América Latina. FLACSO - Chile, Santiago de Chile.

- Fondo Internacional de Emergencia de las Naciones Unidas para la Infancia -Consejo de Educación Secundaria (UNICEF y CES). (2013). Bullying. Experiencias y dimensiones del acoso escolar. UNICEF, Montevideo.

- $\quad$ Fondo Internacional de Emergencia de las Naciones Unidas para la Infancia (UNICEF). (2011). Violencia escolar en América Latina y el Caribe: Superficie y fondo. UNICEF, Panamá.

- García, A. (2010). "Exponiendo hombría. Los circuitos de la hipermasculinidad en la configuración de prácticas sexistas entre varones jóvenes". Revista de Estudios de Juventud, (89), pp. 59 -78.

- Generelo, J. (Coord.) (2012). Acoso escolar homofóbico y riesgo de suicidio en adolescentes y jóvenes LGB. FELGTB -COGAM, Madrid.

- $\quad$ Gómez, A; Gala, F; Lupiani, M; Bernalte, A; Miret, A; Lupiani, S y Barreto, M. (2007). "El bullying y otras formas de violencia adolescente". Cuad. Méd. Forense, 13(48), pp. 165-177.

- Guasch, O. (2007). La crisis de la heterosexualidad. Editorial LAERTES, Barcelona.

- Guzmán, C. y Saucedo, C. (2015). "Experiencias, vivencias y sentidos en torno a la escuela y a los estudios. Abordajes desde las perspectivas de alumnos y estudiantes". Revista Mexicana de Investigación Educativa, 20(67), pp. 1019-1054.

- Jennett, M. (2004). Stand up for us, challenging homophobia in schools. Department of Health, Londres.

- Junqueira, R. (2013). "Pedagogia do armário". A normatividade em ação. Revista Retratos da Escola, 7(13), pp. 481-498.

- López, A. y Gelpi, G. (2015). "La atención psicológica desde una perspectiva de diversidad sexual”. En P. López, F. Forrisi y G. Gelpi (Coord.), Salud y diversidad sexual. Guía para profesionales de la salud. MSP, Montevideo.

- López Amurrio, E. (Coord.). (2013). Homofobia en las aulas 2013. ¿Educamos en la diversidad afectivo sexual? COGAM, Montevideo.

- Meyer, I. (2003). "Prejudice, social stress, and mental health in lesbian, gay, and bisexual populations: Conceptual issues and research evidence". Psychological Bulletin, 129 (5), pp. 674 - 697.

- Ministerio de Desarrollo Social e Instituto Nacional de la Juventud y Adolescencia (MIDES). (2015) Informe. Tercera Encuesta Nacional de Adolescentes y Juventud. MIDES, Montevideo

- Ministerio de Salud Pública (MSP). (2017). Guía para la atención integral de la salud de adolescentes. MSP., Montevideo.

- Observatorio Argentino de Violencia en las Escuelas (2011). la acitírc anU ?aleucse al ne rivivnoc omóC $i$ concepto de bullying. Reflexiones sobre la violencia en la escuela. Ministerio de Educación, Buenos Aires.

- Olavarría, J. (2005). "La masculinidad y los jóvenes adolescentes". Reflexiones pedagógicas. Revista Docencia, 27(10), pp. $46-55$.

- Olweus, D. (2004). "Bullying at school: Prevalence estimation, a useful evaluation design, and a new national initiative in Norway". Association for Child Psychology and Psychiatry Occasional Papers, 23, pp. $5-17$

- $\quad$ Olweus, D. (1993). Bullying at School. What we know and whal we can do. Wiley - Blackwell, Nueva York. 
REVISTA de LA ESCUela de CIENCIAS DE LA EdUCACIÓN, AÑo 15, NRO. 14, VOL. 1, ENERO A JUNIO dE 2019. PÁGINAS 36-44. ISSN 1851-6297 (DESDE DICIEMBRE DE 2006 A DICIEMBRE DE 2017). ISSN 2362-3349 (EN LINEA). SER VICTIMA DE BULLYNG HOMOFÓBICO EN URUGUAY. LAS VOCES DE VARONES ADOLESCENTES DE MONTEVIDEO. GONZALO GELPI.

- $\quad$ Organización de las Naciones Unidas para la Educación, la Ciencia y la Cultura (UNESCO). (2018). Ensinar respeito por todos: Guía de implementaçao. UNESCO, Brasilia.

- Organización de las Naciones Unidas para la Educación, la Ciencia y la Cultura (UNESCO). (2015). El bullying homofóbico y transfóbico en los centros educativos. Taller de sensibilización para su prevención. Guía de facilitación. UNESCO, Santiago de Chile.

- Organización de las Naciones Unidas para la Educación, la Ciencia y la Cultura (UNESCO). (2013). Respuestas del Sector de Educación frente al Bullying Homofóbico. Publicación de la Serie Buenas politicas y prácticas para la educación en VIH y salud. UNESCO, Santiago de Chile.

- Organización de las Naciones Unidas para la Educación, la Ciencia y la Cultura (UNESCO). (2011). Plan CEIBAL in Uruguay. UNESCO, Montevideo.

- $\quad$ Organización de las Naciones Unidas (ONU). (2006). Principios de Yogyakarta sobre la aplicación del Derecho Internacional de Derechos Humanos a las Cuestiones de Orientación Sexual e Identidad de Género. ONU, Ginebra.

- Ovejas Negras y Gay and Lesbian Independent School Teachers Network (GLSEN). (2016). Encuesta Nacional de Clima Escolar en Uruguay 2016. Experiencias de niños, niñas y adolescentes lesbianas, gays, bisexuales y trans en establecimientos educativos. Montevideo, Ovejas Negras.

- $\quad$ Piñuel, I. y Oñate, A. (2006). AVE. Acoso y violencia escolar. TEA Ediciones, Madrid.

- $\quad$ Roth, W. (2014). Towards a theory of experience. Science Education, 98(1), 106-126.

- Ruiz, S. (2009). Diversidad sexual en las aulas. Evitar el bullying o acoso homofóbico. Cuadernos Plural, Badajoz.

- $\quad$ Savin - Williams, R. (2009). La nueva adolescencia homosexual. Ediciones Morata, Madrid.

- Schenck, M. (2014). "Sacando la violencia heteronormativa del armario: Docentes LGB y discriminación". En D. Sempol (Coord.), De silencios y otras violencias. Políticas públicas, regulaciones discriminatorias y diversidad sexual. MIDES, Montevideo.

- Sempol, D. (2013a). De los baños a las calles. Historia del movimiento lésbico, gay, trans uruguayo (19842013). Editorial Sudamericana, Montevideo.

- Sempol, D. (Coord.) (2013b). Políticas públicas y diversidad sexual. Análisis de la heteronormatividad en la vida de las personas y las instituciones. MIDES, Montevideo.

- Viñar, M. (2009). Mundos adolescentes y vértigo civilizatorio. Ediciones Trilce, Montevideo. 